\title{
Deep ultraviolet scanning near-field optical microscopy for the structural analysis of organic and biological materials
}

\author{
AUTHOR(S): \\ Aoki, H; Hamamatsu, T; Ito, S
}

\section{CITATION:}

Aoki, H ... [et al]. Deep ultraviolet scanning near-field optical microscopy for the structural analysis of organic and biological materials. APPLIED PHYSICS LETTERS 2004, 84(3): 356 358

\section{ISSUE DATE:}

2004-01-19

URL:

http://hdl.handle.net/2433/39662

\section{RIGHT:}

Copyright 2004 American Institute of Physics. This article may be downloaded for personal use only. Any other use requires prior permission of the author and the American Institute of Physics. 


\title{
Deep ultraviolet scanning near-field optical microscopy for the structural analysis of organic and biological materials
}

\author{
Hiroyuki Aoki, ${ }^{\text {a) }}$ Toyohiro Hamamatsu, and Shinzaburo Ito \\ Department of Polymer Chemistry, Graduate School of Engineering, Kyoto University, Nishigyo, \\ Kyoto 615-8510, Japan
}

(Received 16 September 2003; accepted 24 November 2003)

\begin{abstract}
Scanning near-field optical microscopy (SNOM) using a deep ultraviolet (DUV) light source was developed for in situ imaging of a variety of chemical species without staining. Numerous kinds of chemical species have a carbon-carbon double bond or aromatic group in their chemical structure, which can be excited at the wavelength below $300 \mathrm{~nm}$. In this study, the wavelength range available for SNOM imaging was extended to the DUV region. DUV-SNOM allowed the direct imaging of polymer thin films with high detection sensitivity and spatial resolution of several tens of nanometers. In addition to the polymer materials, we demonstrated the near-field imaging of a cell without using a fluorescence label. (C) 2004 American Institute of Physics.
\end{abstract}

[DOI: $10.1063 / 1.1642757]$

Scanning near-field optical microscopy (SNOM) has attracted much attention in wide fields of chemical and biological researches, because it enables one to obtain an image by chemical contrast through various spectroscopic techniques with a nanometric spatial resolution. In the measurement by SNOM, fluorescence spectroscopy is most commonly employed. The fluorescence SNOM is so sensitive that it can detect the signal from a single molecule, ${ }^{1-4}$ and provides valuable information such as the orientation and spatial distribution of the fluorescent molecules from the spectra and time-resolved profiles. ${ }^{5,6}$ However, the fluorescence spectroscopy is applicable only to the specimen which is fluorescent or labeled with dye molecules. Recently, several groups have reported Raman scattering spectroscopic techniques in the near-field. ${ }^{7-11}$ Since Raman scattering is associated with the transition between the vibrational energy levels for a chemical bond in a molecule, it allows the direct observation of a variety of chemical species without a staining procedure. However, it is practically difficult to detect the Raman signal by SNOM because of the extremely low scattering efficiency and the small excitation volume illuminated by the localized optical near field. So far only several kinds of chemical species have been observed by Raman SNOM because of the difficulty in the enhancement of the scattering signal up to the intensity level for the high resolution imaging. On the other hand, the fluorescence detection is highly sensitive in comparison to the Raman detection because the cross section of fluorescence is larger by a factor of more than 10 orders compared to that of Raman scattering. Therefore, if the fluorescence SNOM is applicable to the chemical species other than "fluorescent dye," it would become a powerful microscopic technique for chemical mapping with high detection sensitivity and spatial resolution.

In this letter, we describe the near-field fluorescence microscopy using a deep ultraviolet (DUV) light source. Most chemical species are excited by the DUV light below the

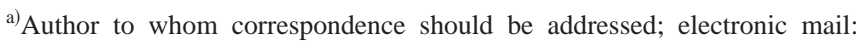
aoki@photo.polym.kyoto-u.ac.jp
}

wavelength of $300 \mathrm{~nm}$. Since many kinds of organic molecules have a conjugated carbon-carbon double bond in their chemical structure, the DUV excitation gives rise to autofluorescence of these molecules in UV wavelengths. For example, the simplest aromatic compound, benzene, has an absorption band around $250 \mathrm{~nm}$ and emits fluorescence at 270-300 nm. The optical near field at the DUV wavelength enables one to directly observe a variety of chemicals with high spatial resolution. Whereas the SNOM imaging at a near UV wavelength range has been reported by several groups, ${ }^{12-14}$ it can be used only for conjugated molecules and the observable species have been still limited. Recently, Sands et al. has reported the resonant Raman SNOM imaging using a DUV light source. ${ }^{11}$ Because of the extremely low signal intensity for the Raman scattering, it took several hours to obtain a SNOM image. With regard to organic or biological samples, the high signal collection efficiency is required in order to reduce an exposure dose from an excitation source and to obtain a micrograph in a short time. The DUV fluorescence SNOM imaging described in this letter is based on the detection of the strong fluorescence signal under the DUV excitation, which is applicable to a variety of chemical species with high sensitivity and subwavelength spatial resolution. In this study, the DUV transmittable probe and signal collection optics were implemented in the SNOM system, and the fluorescence SNOM imaging under DUV excitation was demonstrated for polymer and cell samples without fluorescent labeling.

The SNOM probe is the most crucial part for the DUV operation. The wavelength range available for the conventional SNOM imaging has been limited to the visible region due to the probe. In this study, the probe was made from an optical fiber with a pure quartz core. The fiber was sharpened by a heating-and-pulling process followed by chemical etching in buffered HF. ${ }^{15}$ The quartz SNOM probe was installed to a commercially available scanning probe microscopy system (SP-301A, Unisoku). The laser beam at $266 \mathrm{~nm}$ (fourth harmonic of a diode-pumped Nd:YAG laser) was coupled to the cleaved end of the fiber probe by a single mode fiber 

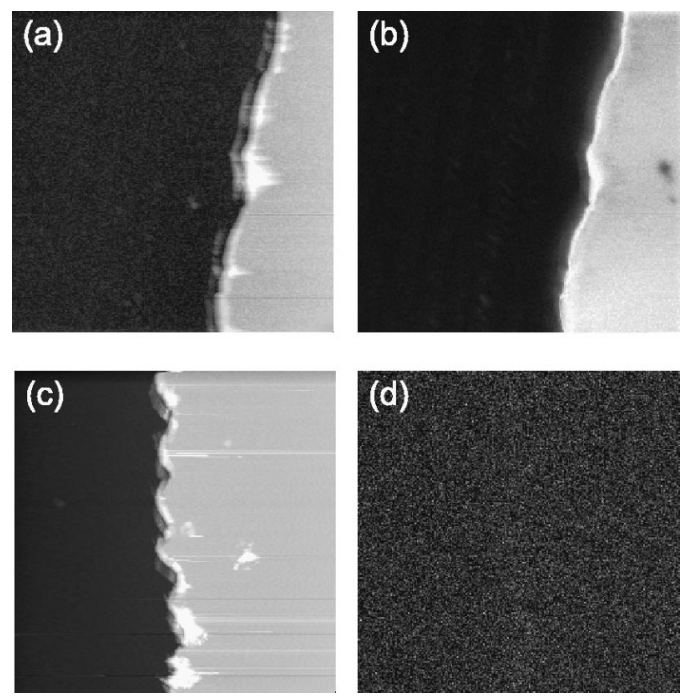

FIG. 1. Topographic and fluorescence SNOM images in the DUV region for PS and PMMA spin cast films: (a) topography and (b) SNOM images for the PS film; (c) topography and (d) SNOM images for PMMA. The left-hand side of each image indicates the substrate surface exposed by scratching the polymer film.

coupler (Newport) equipped with quartz objective and steering lenses. The gap between the probe end and the sample surface was regulated by a shear force feedback mechanism. The ultraviolet fluorescence signal from a specimen was collected with a microscope objective made of quartz (1.25 numerical aperture, $40 \times$, glycerol immersion) and detected by a photomultiplier (R4220P, Hamamatsu Photonics). Optical filters (Omega Optical) were put in front of the detector to block the excitation light.

The polystyrene (PS) and poly(methyl methacrylate) (PMMA) were used as the test samples. The toluene solution of a polymer was spin cast on a quartz cover slip. A part of the sample film was scratched to remove the polymers, and the SNOM measurement was performed for the area including the edge between the polymer and the exposed substrate surfaces. Figure 1 shows the topographic and DUV fluorescence images for the PS and PMMA samples. The right- and left-hand sides of each image indicate the polymer and exposed quartz surfaces, respectively. The thickness of the polymer films was estimated to be $\sim 200 \mathrm{~nm}$ from the height difference between the polymer and substrate surface for each sample. The PS domain was observed as the bright area in the SNOM image obtained by collecting the light at the wavelength from 280 to $350 \mathrm{~nm}$ [Fig. 1(b)]. The fluorescence from the PS thin film excited at $266 \mathrm{~nm}$ showed the maximum intensity at $310 \mathrm{~nm}$, which is attributed to the phenyl ring excimer emission. Thus, the spatial distribution of PS was clearly imaged by the direct detection of the optical signal without any staining procedure. The measured fluorescence intensity from the PS film with a thickness of $200 \mathrm{~nm}$ was 45000 photon counts/s. Since a few hundred photons per second is enough to obtain a clear image, the DUVSNOM has such a high detection sensitivity as to measure the films of aromatic compounds as thin as $1 \mathrm{~nm}$. Similarly to PS, other polymer materials having an aromatic ring such as polyester were clearly observed and characterized from the emission spectra by the DUV excitation. On the other hand, since PMMA does not absorb the light at the excitation Downloaded 11 Jun 2007 to 130.54 .110 .22 . Redistribution subject (a)

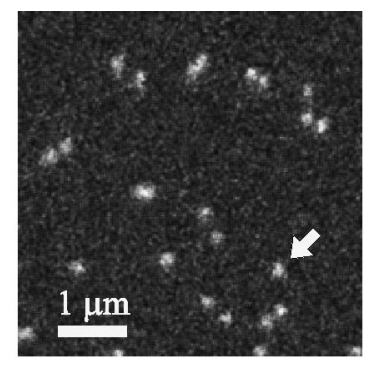

(b)

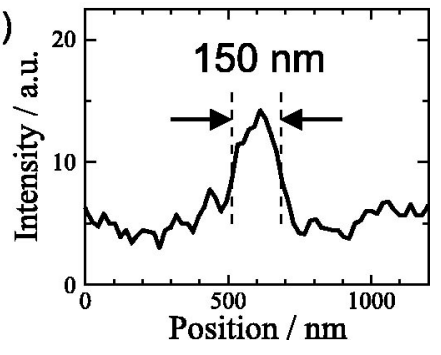

FIG. 2. DUV-SNOM image of "nonfluorescent" PS nanoparticles with a diameter of $100 \mathrm{~nm}$ (a) and the cross section profile for the PS particle indicated by the arrow in the fluorescence image (b). The sample was prepared by spin coating from a PS latex dispersion in a 4 wt \% poly(vinyl alcohol) solution.

wavelength of $266 \mathrm{~nm}$, there is no contrast in the SNOM image [Fig. 1(d)]. Since PMMA has an absorption band attributed to electronic transition of the carbonyl group, it can be observed in the SNOM image by adjusting the excitation wavelength.

In order to demonstrate the spatial resolution beyond the diffraction limit, we performed the measurement of the PS latex beads with a diameter of $100 \mathrm{~nm}$ dispersed on a quartz substrate. The PS beads are commercially available as the "nonfluorescent" latex (Polybead carboxylate, Polyscience). Figure 2(a) depicts the fluorescence SNOM image of the PS nanoparticles. Bright fluorescence was detected from each bead with a volume on the order of attoliter; the SNOM image was taken within $15 \mathrm{~min}$. The cross section profile for a PS particle is shown in Fig. 2(b). The particle was observed as the fluorescence spot with a diameter of $150 \mathrm{~nm}$ in the DUV-SNOM image, that is, the particle was broadened by $50 \mathrm{~nm}$. This indicates that the point spread function is about $50 \mathrm{~nm}$, which is beyond the diffraction limit.

The DUV-SNOM imaging is applicable not only to polymer materials but also to biological samples. Since nucleic acids and part of the natural amino acids have conjugated functional groups, they absorb the light in the ultraviolet region and emit the fluorescence at $300-400 \mathrm{~nm}$. This indicates that DUV-SNOM can be used for imaging biomaterials such as protein and DNA. Figure 3 shows the DUVSNOM image of the stromal cell, PA6. The fibrous structure under the cell membrane can be clearly seen in the SNOM image with a spatial resolution of $150 \mathrm{~nm}$. At the excitation wavelength of $266 \mathrm{~nm}$, it is thought that all of the protein and DNA in the cell were excited and emit the fluorescence in this image. Since the SNOM developed in this study enables us to use a broad wavelength range from DUV to near infrared, the selective imaging of a particular site is possible by the different excitation/collection wavelengths much wider than the conventional SNOM in the visible region.

In summary, the wavelength range of the excitation 


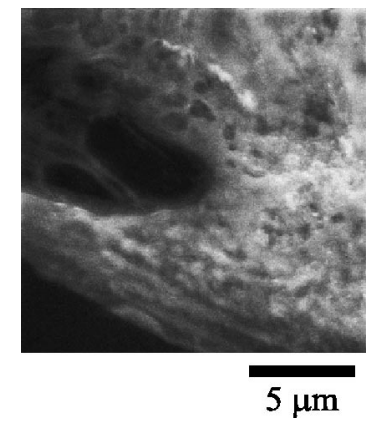

FIG. 3. SNOM image of the stromal cell, PA6. The excitation and detection wavelengths were 266 and $280-360 \mathrm{~nm}$, respectively. The scale bar indicates $5 \mu \mathrm{m}$.

source of SNOM was extended to the deep ultraviolet region by the DUV transmittable probe and signal collection optics. By illuminating the specimens with the optical near field in the DUV region, most kinds of chemical compounds having aromatic substituents can be directly imaged through the fluorescence emission. The near-field imaging was demonstrated for thin films of polymer and biological materials without staining, and the spatial resolution of $50 \mathrm{~nm}$ was achieved. Moreover, the DUV-SNOM has high sensitivity to observe ultrathin films with a nanometric thickness because of the contrast mechanism of fluorescence detection. DUV-SNOM allows the direct observation of a variety of chemical species with a high resolution and sensitivity, and it will be a powerful microscopic technique in wide research fields.
The authors would like to thank Professor Hiroo Iwata and Professor Koichi Kato, Institute for Frontier Medical Sciences, Kyoto University, for the preparation of the cell samples. This work was supported by Konica Imaging Science Foundation and a Grant-in-Aid from the Ministry of Education, Culture, Sports, Science, and Technology of Japan.

${ }^{1}$ E. Betzig and R. Chichester, Science 262, 1422 (1993).

${ }^{2}$ C. W. Hollars and R. C. Dunn, J. Phys. Chem. 112, 7822 (2000).

${ }^{3}$ N. F. van Hulst, J. A. Veerman, M. F. Garcia-Parajo, and L. Kuipers, J. Chem. Phys. 112, 7799 (2000).

${ }^{4}$ F. Vargas, O. Hollricher, O. Marti, G. de Schaetzen, and G. Tarrach, J. Chem. Phys. 117, 866 (2002).

${ }^{5}$ H. Aoki, S. Tanaka, S. Ito, and M. Yamamoto, Macromolecules 33, 9650 (2000).

${ }^{6}$ H. Aoki and S. Ito, J. Phys. Chem. B 105, 4558 (2001).

${ }^{7}$ R. M. Stockle, Y. D. Suh, V. Deckert, and R. Zenobi, Chem. Phys. Lett. 318, 131 (2000)

${ }^{8}$ N. Hayazawa, Y. Inouye, Z. Sekkat, and S. Kawata, Chem. Phys. Lett. 335, 369 (2001).

${ }^{9}$ N. Hayazawa, Y. Inouye, Z. Sekkat, and S. Kawata, J. Chem. Phys. 117, 1296 (2002)

${ }^{10}$ A. Hartschuh, E. J. Sanchez, S. Xie, and L. Novotny, Phys. Rev. Lett. 90, 095503 (2003).

${ }^{11}$ H. S. Sands, F. Demangeot, E. Bonera, S. Webster, R. Bennett, I. P. Hayward, F. Marchi, D. A. Smith, and D. N. Batchelder, J. Raman Spectrosc. 33, 730 (2002).

${ }^{12}$ H. Aoki, Y. Sakurai, S. Ito, and T. Nakagawa, J. Phys. Chem. B 103, 10553 (1999).

${ }^{13}$ R. Stevenson, R. Riehn, R. G. Milner, D. Richards, E. Moons, D. J. Kang, M. Blamire, J. Morgado, and F. Cacialli, Appl. Phys. Lett. 79, 833 (2001).

${ }^{14}$ J. Chappell and D. G. Lidzey, J. Microsc. 209, 188 (2003).

${ }^{15}$ M. Ohtsu, Near-Field Nano/Atom Optics and Technology (Springer, Tokyo, 1998). 